\title{
Anaesthesia Concept as Simple as Possible
}

\author{
Alaa Ali M Elzohry*, Mohammed Farghaly Abed Elhamed, Mohanad AM, Abed El Gawad and Bahaa \\ Gamal Saad \\ Lecturer of Anesthesia, Assiut University, Egypt
}

Received: June 16, 2018; Published: June 22, 2018

*Corresponding author: Alaa Ali M Elzohry, Lecturer of Anesthesia, Assiut University, Egypt

\begin{abstract}
When you meet your anesthesia provider prior to surgery, you're about to trust your life to a stranger. It matters who that stranger is? As a patient, do you have any control over who your anesthesia provider will be? If your surgery is an emergency at 2 a.m. when only one anesthesia provider is available, you will not. But for most surgeries, and all elective surgeries, you have choices. General or regional anesthesia, will you choose specially if you have major medical problems that restrict your life activity? Currently, there are significant advances in anesthetic techniques for many common surgeries. The objective of this review is to demonstrate the benefits, risks and the use of the different anesthetic techniques as well as a how to choose the ideal technique suitable for your surgery.
\end{abstract}

Keywords: General Anesthesia; Regional Anesthesia; Thoracic Surgery; Upper Abdominal Surgery Awareness.

\section{Introduction}

If we ask our self about Medicine and its progress, only few events came to his mind. Antimicrobial therapy, vaccinations, surgical therapy of cancer and continuous surgical innovations, to cite only some instances, did ameliorate human health. But most of the people forget that many surgical innovations and the care of a lot of infectious disease were due to the birth and upgrading of an often neglected branch of Medicine: Anesthesia and Intensive Care. Furthermore, if I ask to any person, who has to undergo a surgical procedure what is his main trouble, I'm reasonably sure that his answer will be: "I'm afraid of anesthesia". This fear derives from the loss of both self-control and consciousness due to anesthetic administration. When doctors have become aware of the problem and their studies started to include anesthesia, the anesthesiologist stopped to be a kind of magician and became a professional figure among the other specialized doctors. The practice of modern surgery and anesthesia is becoming even more scientific and evidencebased. Students will be encouraged to critically evaluate scientific evidence from primary sources. Is the practice of anesthesia an art or a science?

Is the practice of medicine an art or a science? Over one hundred years ago the fathemodern medicine, Dr. William Osler of Johns Hopkins Medical Center, made the following statements: "Medicine is a science of uncertainty and an art of probability," and "The practice of medicine is an art, based on science."Attempts at producing a state of general anesthesia can be traced throughout recorded history in the writings of the ancient Sumerians, Babylonians, Assyrians, Egyptians, Greeks, Romans, Indians, and Chinese.
During the Middle Ages, scientists and other scholars made significant advances in the Eastern world, while their European counterparts also made important advances.The European Renaissance saw significant advances in anatomy and surgical technique. However, despite all this progress, surgery remained a treatment of last resort. Largely because of the associated pain, many patients chose certain death rather than undergo surgery.

Two enormous leaps occurred in the late 19th century, which together allowed the transition to modern surgery. Concurrent with these developments were the significant advances in pharmacology and physiology which led to the development of general anaesthesia and the control of pain. On the 14 November 1804, Hanaoka Seishū, a Japanese doctor, became the first person to successfully perform surgery using general anaesthesia. In the 20th century, the safety and efficacy of general anaesthesia was improved by the routine use of tracheal intubation and other advanced airway management techniques. Significant advances in monitoring and new anaesthetic agents with improved pharmacokinetic and pharmaco dynamic characteristics also contributed to this trend. Finally, standardized training programs for anaesthesiologists and nurse anaesthetists emerged during this period. When you have surgery, do you care who administers your anesthetic? You should. An oft-repeated medical adage states: "anesthesia is $99 \%$ boredom and 1\% panic."

As an anesthesiologist who's delivered over 50,000 hours of operating room care over 25 years, I can attest that the adage is true. Ninety-nine percent of the time, the anesthesia provider's job requires vigilance during a patient's stable progression of metro- 
nome heartbeats and regular breathing, but $1 \%$ of the time requires clear thinking and prompt action during moments of sheer panic. These stress-filled episodes of panic are unknown to the general public, yet represent ordeals that every anesthesia provider must rise above to protect their patients. Webster's Dictionary defines panic as "an overwhelming feeling of fear and anxiety." If you were to observe an anesthesiologist at work, you would see little or no evidence of overwhelming fear or anxiety. Even under dire emergencies, most anesthesia providers remain outwardly composed and efficient while they make the necessary diagnoses and apply the appropriate treatments. But anesthesiologists are human-no human can watch another human trying to die without feeling intense emotions. These emotions are fear and anxiety. The operating room is an intense environment. Operating room medicine is pressure-packed for four reasons:

a) Anesthetic drugs change the physiology of patients in profound ways.

b) Surgeons do dangerous things to patients.

c) Surgical patients have diseases. Some of these diseases are urgent or severe.

d) Human beings make errors. This includes both surgeons and anesthesia providers.

Unbelievable events occur at unexpected times in operating rooms, and your anesthesia provider must keep you safe. He or she is in control of your airway, breathing, and circulation at every moment. Your anesthesia provider is your insurance policy against medical complications during surgery. Your anesthesia provider's job is to play Goalie at the Pearly Gates, and keep you alive. The individual administering your anesthesia can vary-your anesthesia provider may be:

\section{a) A medical doctor (an anesthesiologist)}

b) A certified registered nurse anesthetist (CRNA) or anesthesia assistant (AA) supervised by an anesthesiologist

c) A CRNA working without anesthesiologist supervision.

When you meet your anesthesia provider prior to surgery, you're about to trust your life to a stranger. It matters who that stranger is. As a patient, do you have any control over who your anesthesia provider will be? If your surgery is an emergency at 2 a.m. when only one anesthesia provider is available, you will not. But for most surgeries, and all elective surgeries, you have choices. When I describe the elation of interacting with anesthesia patients, my best friend offers a simple explanation: "Of course your patients respect you before the surgery. You're about to knock them unconscious. They'll have no control and they're completely dependent on you. They want you to like them. They want you to keep them alive." I believe that assessment is accurate. Every patient wants the same thing from their anesthesia provider. A successful, complication-free experience. And that's what happens almost every time. I commonly hear two questions from my patients: "How does anesthesia work?" and "How do the anesthetic drugs make me fall asleep?"The short answer to both questions is, "We're not sure."
This column is designed as a brief tutorial for non-anesthesiologists who wish to better comprehend how anesthetic drugs work. General anesthesia is the sum of hypnosis (sleep), amnesia, analgesia (pain relief), and the lack of any motion response to pain. Propofol and barbiturates cause hypnosis. Versed and other benzodiazepines cause amnesia. Narcotics such as morphine and fentanyl cause analgesia. Paralyzing drugs such as rocuronium, vecuronium and succinylcholine cause muscle relaxation and lack of motion. The potent inhaled anesthetics sevoflurane, desflurane, and isoflurane produce all four of the effects of hypnosis, amnesia, analgesia, and lack of motion. Drugs such as propofol and Versed are injected into the bloodstream and are circulated to the central nervous system, where they carry out their effect on brain cells. Inhaled anesthetics such as sevoflurane and desflurane traverse from the lungs into the bloodstream and are circulated to the central nervous system, where they carry out their effect on brain cells.

So, General anaesthesia has many purposes, including:

a) Analgesia (loss of response to pain)

b) Amnesia (loss of memory)

c) Immobility (loss of motor reflexes)

d) Hypnosis (unconsciousness)

e) Paralysis (skeletal muscle relaxation)

Narcotics such as morphine, fentanyl \&Demerol cause pain relief by binding to opioid receptors in the brain (or the spinal cord). The most common narcotic side effects, e.g. sleepiness and nausea, also arise from the direct effect of the narcotics on the brain. Modern anesthetic care can involve all the drugs discussed above. For example, in a general anesthetic for an abdominal surgery, the anesthesiologist may inject Versed into the IV as a premedication to reduce anxiety, then inject propofol into the IV to initiate sleep, and inject rocuronium into the IV to induce muscle relaxation/paralysis prior to inserting an endo tracheal breathing tube. General anesthesia is then maintained by the administration of a potent inhaled anesthetic gas such as sevoflurane, supplemented by the intravenous injection of a narcotic such as fentanyl to assure post-operative pain relief. The selection of the proper anesthetic drugs and doses for each individual patient makes anesthesiology both fun and fascinating.

\section{Premedication}

Prior to administration of a general anaesthetic, the anaesthetist may administer one or more drugs that complement or improve the quality or safety of the anaesthetic. One commonly used premedication is clonidine, an alpha-2 adrenergic agonist [1]. Clonidine premedication reduces the need for anaesthetic induction agents, for volatile agents to maintain general anaesthesia, and for postoperative analgesics [2]. It also reduces postoperative shivering, postoperative nausea and vomiting, and emergence delirium. In children, clonidine premedication is at least as effective as benzodiazepines and has less serious side effects [3]. Midazolam, a benzodiazepine is effective in reducing preoperative anxiety, including separation anxiety in children [4]. Dexmedetomidine and certain 
atypical antipsychotic agents may be used in uncooperative children [5]. Melatonin has been found to be effective as an anaesthetic premedication in both adults and children because of its hypnotic, anxiolytic, sedative, antinociceptive, and anticonvulsant properties. Unlike midazolam, melatonin does not impair psychomotor skills or hinder recovery. Recovery is more rapid after premedication with melatonin than with midazolam, and there is also a reduced incidence of post-operative agitation and delirium [6].

Another example of anaesthetic premedication is the preoperative administration of beta adrenergic antagonists to reduce the incidence of postoperative hypertension, cardiac dysrhythmia, or myocardial infarction. Anaesthesiologists may administer an antiemetic agent such as ondansetron, droperidol, or dexamethasone to prevent postoperative nausea and vomiting, or subcutaneous heparin or enoxaparin to reduce the incidence of deep vein thrombosis. Other commonly used premedication agents include opioids

such as fentanyl or sufentanil, gastrokinetic agents such as metoclopramide, and histamine antagonists such as famotidine [6]. Non-pharmacologic preanaesthetic interventions include playing relaxing music, massage, and reducing ambient light and noise levels in order to maintain the sleep-wake cycle [7]. Minimizing sensory stimulation or distraction by video games may help to reduce anxiety prior to or during induction of general anaesthesia [7]. Parental presence during premedication and induction of anaesthesia has not been shown to reduce anxiety in children [8]. It is suggested that parents who wish to attend should not be actively discouraged, and parents who prefer not to be present should not be actively encouraged to attend [8].

\section{Stages of Anaesthesia}

Guedel's classification, introduced by Arthur Ernest Guedel in 1937 [9]. Describes four stages of anaesthesia.

Stage 1: Stage 1, also known as induction, is the period between the administration of induction agents and loss of consciousness. During this stage, the patient progresses from analgesia without amnesia to analgesia with amnesia. Patients can carry on a conversation at this time.

Stage 2: Stage 2, also known as the excitement stage, is the period following loss of consciousness and marked by excited and delirious activity. During this stage, the patient's respiration and heart rate may become irregular. In addition, there may be uncontrolled movements, vomiting, suspension of breathing, and pupillary dilation. Because the combination of spastic movements, vomiting, and irregular respiration may compromise the patient's airway, rapidly acting drugs are used to minimize time in this stage and reach Stage 3 as fast as possible.

Stage 3: In Stage 3, also known as surgical anaesthesia, the skeletal muscles relax, vomiting stops, respiratory depression occurs, and eye movements slow and then stop. The patient is unconscious and ready for surgery.

Stage 4: Stage 4, also known as overdose, occurs when too much anaesthetic medication is given relative to the amount of sur- gical stimulation and the patient has severe brainstem or medullary depression, resulting in a cessation of respiration and potential cardiovascular collapse. This stage is lethal without cardiovascular and respiratory support. General anesthesia is different from conscious sedation, which may be used for some diagnostic procedures, such as radiology examinations, when the patient can easily be awakened by a mild stimulation like calling his or her name.

\section{Physiologic Monitoring}

Several monitoring technologies allow for a controlled induction of, maintenance of, and emergence from general anaesthesia [10].

Continuous Electrocardiography (ECG or EKG): Electrodes are placed on the patient's skin to monitor heart rate and rhythm. This may also help the anaesthesiologist to identify early signs of heart ischaemia.

Continuous Pulse Oximetry ( $\mathbf{S P O}_{2}$ ): A device is placed, usually on a finger, to allow for early detection of a fall in a patient's haemoglobin saturation with oxygen (hypoxaemia).

Blood Pressure Monitoring: There are two methods of measuring the patient's blood pressure. The first, and most common, is non-invasive blood pressure (NIBP) monitoring. This involves placing a blood pressure cuff around the patient's arm, forearm, or leg. A machine takes blood pressure readings at regular, preset intervals throughout the surgery. The second method is invasive blood pressure (IBP) monitoring. This method is reserved for patients with significant heart or lung disease, the critically ill, and those undergoing major procedures such as cardiac or transplant surgery, or when large blood loss is expected. It involves placing a special type of plastic cannula in an artery, usually in the wrist (radial artery) or groin (femoral artery).

Capnography: measures the amount of carbon dioxide exhaled by the patient in percent or $\mathrm{mmHg}$, allowing the anaesthesiologist to assess the adequacy of ventilation. MmHg is usually used to allow the provider to see more subtle changes.

Temperature Measurement: to discern hypothermia or fever, and to allow early detection of malignant hyperthermia.

Electroencephalography, Entropy Monitoring: or other systems may be used to verify the depth of anaesthesia. This reduces the likelihood of anaesthesia awareness and of overdose.

\section{Airway Management}

Anaesthetized patients lose protective airway reflexes (such as coughing) (Figure 1), airway patency, and sometimes a regular breathing pattern due to the effects of anaesthetics, opioids, or muscle relaxants. To maintain an open airway and regulate breathing, some form of breathing tube is inserted after the patient is unconscious. To enable mechanical ventilation, an endotracheal tube is often used, although there are alternative devices that can assist respiration, such as face masks or laryngeal mask airways. Generally, full mechanical ventilation is only used if a very deep state of general anaesthesia is to be induced for a major procedure, and/ 
or with a profoundly ill or injured patient. That said, induction of general anaesthesia usually results in apnea and requires ventillation until the drugs wear off and spontaneous breathing starts. Spontaneous ventilation has been traditionally maintained with inhalational agents (i.e. halothane or sevoflurane) which is called a gas or inhalational induction. Spontaneous ventilation can also be maintained using intravenous anaesthesia (e.g. propofol). Intravenous anaesthesia to maintain spontaneous respiration has certain advantages over inhalational agents (i.e. suppressed laryngeal reflexes) however it requires careful titration. Spontaneous respiration using Intravenous anaesthesia and high-flow nasal oxygen is a technique that has been used in difficult and obstructed airways [10].

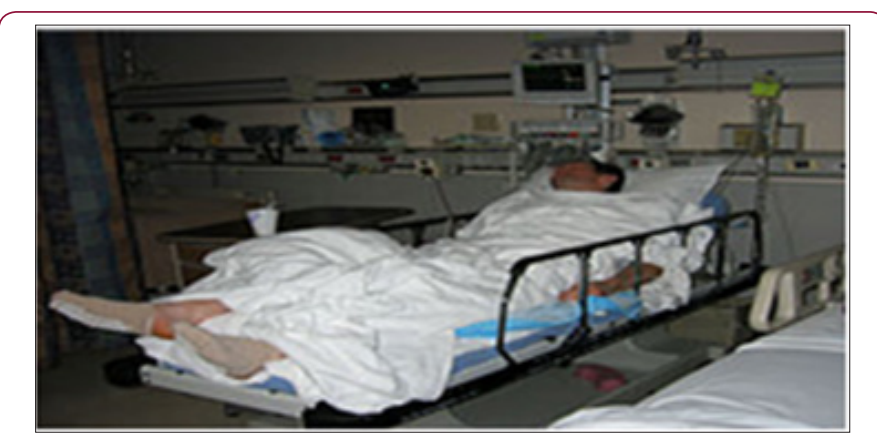

Figure 1: Anaesthetized patient in postoperative recovery.

\section{Eye Management}

General anaesthesia reduces the tonic contraction of the orbicularis oculi muscle, causing lagophthalmos, or incomplete eye closure, in 59\% of patients [11]. In addition, tear production and tear-film stability are reduced, resulting in corneal epithelial drying and reduced lysosomal protection. The protection afforded by Bell's phenomenon (in which the eyeball turns upward during sleep, protecting the cornea) is also lost. Careful management is required to reduce the likelihood of eye injuries during general anaesthesia [12].

\section{Neuromuscular Blockade}

Paralysis, or temporary muscle relaxation with a neuromuscular blocker, is an integral part of modern anaesthesia. The first drug used for this purpose was curare, introduced in the 1940s, which has now been superseded by drugs with fewer side effects and, generally, shorter duration of action. Muscle relaxation allows surgery within major body cavities, such as the abdomen and thorax, without the need for very deep anaesthesia, and also facilitates endotracheal intubation. Paralysis of the muscles of respiration-the diaphragm and intercostal muscles of the chest, requires that some form of artificial respiration be implemented. Because the muscles of the larynx are also paralysed, the airway usually needs to be protected by a tube. Paralysis is most easily monitored by means of a peripheral nerve stimulator.

\section{Maintenance}

The duration of action of intravenous induction agents is generally 5 to 10 minutes, after which spontaneous recovery of consciousness will occur. In order to prolong unconsciousness for the required duration (usually the duration of surgery), anaesthesia must be maintained. This is achieved by allowing the patient to breathe a carefully controlled mixture of oxygen, sometimes nitrous oxide, and a volatile anaesthetic agent, or by administering medication (usually propofol) through an intravenous catheter. Inhaled agents are frequently supplemented by intravenous anaesthetics, such as opioids (usually fentanyl or a fentanyl derivative) and sedatives (usually propofol or midazolam). At the end of surgery, administration of anaesthetic agents is discontinued. Recovery of consciousness occurs when the concentration of anaesthetic in the brain drops below a certain level .Other medications are occasionally used to treat side effects or prevent complications. They include antihypertensives to treat high blood pressure; ephedrine or phenylephrine to treat low blood pressure; salbutamol to treat asthma, laryngospasm, or bronchospasm; and epinephrine or diphenhydramine to treat allergic reactions. Glucocorticoids or antibiotics are sometimes given to prevent inflammation and infection, respectively [13].

\section{Emergence}

Emergence is the return to baseline physiologic function of all organ systems after the cessation of general anaesthetics. This stage may be accompanied by temporary neurologic phenomena, such as agitated emergence (acute mental confusion), aphasia (impaired production or comprehension of speech), or focal impairment in sensory or motor function. Shivering is also fairly common and can be clinically significant because it causes an increase in oxygen consumption, carbon dioxide production, cardiac output, heart rate, and systemic blood pressure. A number of techniques are used to reduce shivering, such as increased ambient temperature, conventional or forced warm air blankets, and warmed intravenous fluids [14].

\section{Postoperative Care}

Anaesthesia should conclude with a pain-free awakening and a management plan for postoperative pain relief. This may be in the form of regional analgesia or oral, transdermal, or parenteral medication. Minor surgical procedures are amenable to oral pain relief medications such as paracetamol and NSAIDs (e.g., ibuprofen). Moderate levels of pain require the addition of mild opiates such as tramadol. Major surgical procedures may require a combination of modalities to confer adequate pain relief. Parenteral methods include patient-controlled analgesia (PCA) involving a strong opioid such as morphine, fentanyl, or oxycodone [15].

\section{Perioperative Mortality}

Most perioperative mortality is attributable to complications from the operation, such as haemorrhage, sepsis, and failure of vital organs. Current estimates of perioperative mortality in procedures involving general anaesthesia range from one in 53 to one in 5,417 [16-17]. Mortality directly related to anaesthetic management is very uncommon but may be caused by pulmonary aspiration of gastric contents [18] asphyxiation [19] or anaphylaxis [20]. These in turn may result from malfunction of anaesthesia-related equipment or, more commonly, human error. A 1978 study found that $82 \%$ of preventable anaesthesia mishaps were the result of human error [21]. In 1984, American anaesthesiologist Ellison C. Pierce ap- 
pointed the Anesthesia Patient Safety and Risk Management Committee within the American Society of Anesthesiologists [22]. This committee was tasked with determining and reducing the causes of anaesthesia-related morbidity and mortality [22]. An outgrowth of this committee, the Anesthesia Patient Safety Foundation, was created in 1985 as an independent, nonprofit corporation with the goal "that no patient shall be harmed by anesthesia" [23].

\section{Anesthesia Awareness}

It is the phenomenon of a patient having general awareness so they can recall events that occurred during his/her surgery. Occasionally patients remember voices, machines beeping or, very rarely, may feel pain at the surgery site. Some patients try to signal that they are awake, but if muscle paralysis is required for the surgery, movement is not possible. This was the premise of the movie "Awake," which was released last fall.

Awareness under anesthesia for surgery is uncommon, with an incidence in the United States of roughly one patient per 700 . Yet every case is a cause for concern. This is especially so when an exaggerated fear of the risk of awareness under anesthesia results in a patient not choosing surgery when it may be the best medical option. Awareness during general anesthesia may occur at the start of anesthesia, when anesthetics have not taken full effect, or at the end of surgery when the patient is waking up after anesthesia has been stopped. It is only very rarely that this occurs during surgery itself. However affected individuals may suffer significant psychological trauma from such an event. A number of reasons exist why awareness under anesthesia for surgery can happen. Deep anesthesia can be dangerous in some high-risk surgery patients. This could be the case for people undergoing heart surgery, trauma surgery or in critically ill patients requiring any surgical procedure. Furthermore the dosage requirements for anesthetic drugs to keep a patient unconscious differ from person to person, and each person reacts differently to anesthesia [24]. Another reason for awareness under anesthesia may be from human error, such as giving the wrong medication or a technical failure in the equipment used to deliver anesthesia.

\section{Minimizing Anesthesia Awareness}

Anesthesia providers are critically aware of this potential problem. There is no substitute for experience, expertise and training as the best way to minimize awareness during the procedure requiring general anesthesia. Since there is no single, reliable monitor of this phenomenon, judgment needs to be used in the administration of anesthetics to minimize unwanted effects [25]. There is developing evidence that, in some categories of patients at high risk of awareness, a certain type of brainwave monitoring may help decrease the incidence of awareness. Such monitors can be used as an additional way of assessing anesthetic depth, but they are not a solution in themselves [26].

\section{What Patients Can Do?}

It is crucial for patients to discuss concerns about awareness and any other issue related to anesthesia during the preoperative visit or interview with an anesthesiologist. The best choice of anesthesia for the particular surgery can then be discussed. In the unlikely situation that a patient thinks he or she was awake during surgery, it is very important to talk to the anesthesia provider about the experience. Doing so will help establish when awareness may have occurred and allow the anesthesia provider to explain events in the operating room that could have led to awareness. It also may be important to provide psychological counseling to some patients who have experienced awareness under anesthesia. The American Society of Anesthesiologists and the American Society of Nurse Anesthetists understand patients fear the possibility of awareness and provide an information booklet on this subject listing 10 points that surgical patients should know. This is readily accessible at www. asahq.org/patientEducation.htm\#awareness and www.aana.com. One of the most common questions I hear from patients immediately prior to their surgical anesthetic is, "Will I have a breathing tube down my throat during anesthesia?"he answer is: It depends .Let's answer this question for some common surgeries: Anesthetic options include general anesthesia, regional anesthesia, or local anesthesia.

Nose and Throat Surgeries such as Tonsillectomy and Rhinoplasty: Almost all nose and throat surgeries require an airway tube, so anesthetic gases and oxygen can be ventilated in and out through your windpipe safely during the time the surgeon is working on these breathing passages.

Abdominal Surgeries, Including Laparoscopy: Almost all intra-abdominal surgeries require an airway tube to guarantee adequate ventilation of anesthetic gases and oxygen in and out of your lungs while the surgeon works inside your abdomen.

Chest Surgeries and Open Heart Surgeries: Almost all intra-thoracic surgeries require an airway tube to guarantee adequate ventilation of anesthetic gases and oxygen in and out of your lungs while the surgeon works inside your chest.

Total knee Replacement and Total Hip Replacement: The majority of total knee and hip replacement surgeries are performed using spinal, epidural and/or nerve block anesthesia anesthesia to block pain to the lower half of the body. The anesthesiologist often chooses to supplement the regional anesthesia with intravenous sedation, or supplement with a general anesthetic which requires an airway tube. Why add sedation or general anesthesia to the regional block anesthesia? It's simple: most patients have zero interest in being awake while they listen to the surgeon saw through their knee joint or hammer their new total hip into place.

Cataract Surgery: Cataract surgery is usually performed using numbing local anesthetic eye drop medications. Patients are wake or mildly sedated, and no airway tube is used.

Colonoscopy or Stomach Endoscopy: These procedures are performed under intravenous sedation and almost never require an airway tube.

Hand or Foot Surgeries: The anesthesiologist will choose the simplest anesthetic that suffices. Sometimes the choice is local anesthesia, with or without intravenous sedation. Sometimes the choice will be a regional nerve block to numb the extremity, with or without intravenous sedation. Many times the choice will be a 
general anesthetic, often with an airway tube. An LMA is used more frequently than an ETT.

Cesarean Section: The preferred anesthetic is a spinal or epidural block which leaves the mother awake and alert to bond with her newborn immediately after childbirth. If the Cesarean section is an urgent emergency performed because of maternal bleeding or fetal distress, and there is inadequate time to insert a spinal or epidural local anesthetic into the mother's lower back, a general anesthetic will be performed. An ETT is always used.

Pediatric Surgeries: Tonsillectomies are a common procedure and require a breathing tube as described above. Placement of pressure ventilation tubes into a child's ears requires general anesthetic gases to be delivered via facemask only, and no airway tube is required. Almost all pediatric surgeries require general anesthesia. Infants, toddlers, and children need to be unconscious during surgery, for emotional reasons, because their parents are not present. The majority of pediatric general anesthetics require an airway tube.

\section{Spinal Anaesthesia}

Also called intrathecal block [27] is a form of regional anaesthesia involving the injection of a local anaesthetic into the subarachnoid space, generally through a fine needle. The tip of the spinal needle has a point or small bevel. The word subarachnoid translates to "below the arachnoid". The arachnoid is one of the layers of the meninges covering the nerves of the spinal column. In the subarachnoid space lies the cerebral spinal fluid (CSF) which surrounds the spinal cord and brain. In a spinal anesthetic, the subarachnoid space is located with a needle by the anesthesiologist, and the appropriate anesthetic medications are injected. Local anesthetics, such as lidocaine or bupivicaine (brand name Marcaine), given into the subarachnoid space, bring on sensory and motor numbness. The anesthesiologist chooses the dose and type of drug depending on the patient's age, size, height, medical condition, and the type of surgery. Following the onset of numbness from spinal anesthesia, the patient may either stay awake for surgery, or more often intravenous anesthesia is given to achieve a light sleep. Sometimes light general anesthesia is given to supplement spinal anesthesia [28] (Figure 2). Medical uses:

a) Orthopaedic surgery on the pelvis, hip, femur, knee, tibia, and ankle, including arthroplasty and joint replacement

b) Vascular surgery on the legs

c) Endovascular aortic aneurysm repair

d) Hernia (inguinal or epigastric)

e) Haemorrhoidectomy

f) Nephrctomy and cystectomy in combination with general anaesthesia

g) TURP \& TURT

h) Hysterectomy in different techniques used \& Caesarean sections i) Pain management during vaginal birth and delivery.

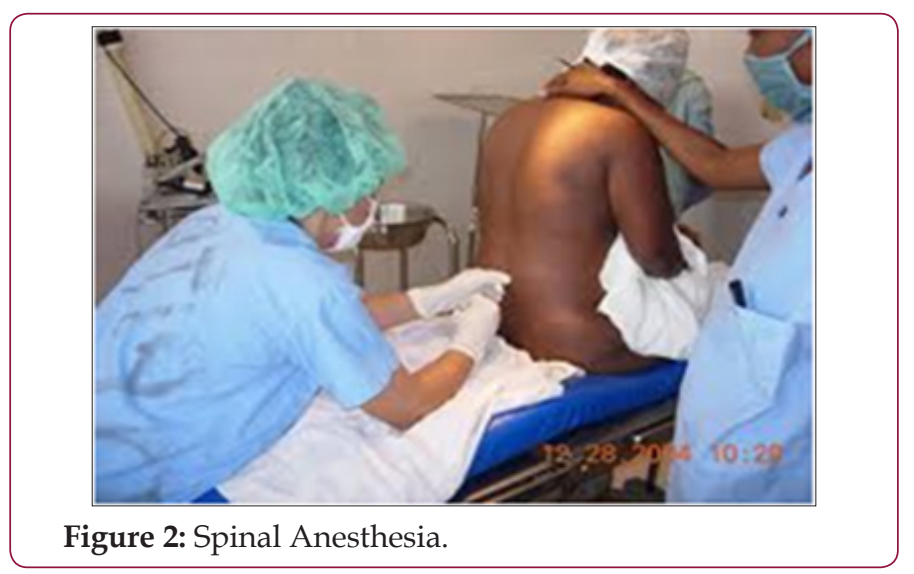

Spinal anaesthesia is the technique of choice for Caesarean section as it avoids a general anaesthetic and the risk of failed intubation .It also means the mother is conscious and the partner is able to be present at the birth of the child. The post operative analgesia from intrathecal opioids in addition to non-steroidal anti-inflammatory drugs is also good. If surgery allows, spinal anaesthesia is very useful in patients with severe respiratory disease such as COPD as it avoids intubation and ventilation. It may also be useful in patients where anatomical abnormalities may make tracheal intubation very difficult [29].

Contraindications:

a) Non-availability of patient's consent.

b) Local infection or sepsis at the site of lumbar puncture.

c) Bleeding disorders, thrombocytopaenia, or systemic anticoagulation (secondary to an increased risk of a spinal epidural hematoma)

d) Space occupying lesions of the brain.

e) Anatomical disorders of the spine.

f) Hypovolaemia e.g. following massive haemorrhage, including in obstetric patients.

g) Risks and complications.

h) Can be broadly classified as immediate (on the operating table) or late (in the post-anaesthesia care unit or ward).

i) Hypotension (neurogenic shock).

j) Post-dural-puncture headache or post-spinal headache Associated with the size and type of spinal needle used.

k) Cardiac arrest - very rare, usually related to the underlying medical condition of the patient.

l) Spinal canal haematoma, with or without subsequent neurological sequelae due to compression of the spinal nerves.

m) Epidural abscess, again with potential permanent neurological damage. May present as meningitis or an abscess with back pain, fever, lower limb neurological impairment and loss of bladder/bowel function. Society and culture. 
Current usage of this technique is waning in the developed world, with epidural analgesia or combined spinal-epidural anaesthesia emerging as the techniques of choice where the cost of the disposable 'kit' is not an issue. However spinal analgesia is the mainstay of anaesthesia in India, Pakistan, and parts of Africa, excluding the major centers. Thousands of spinal anaesthetics are administered daily in hospitals and nursing homes. At a low cost, a surgery of up to two hours duration can be performed below the umbilicus of the patient [30].

\section{Epidural Anesthesia}

Epidural anesthesia is done by the injection of local anesthetic solution, with or without a narcotic medication, into the low back into the epidural space. Prior to beginning anesthesia, the anesthesiologist places monitors of blood pressure, electrocardiogram, pulse and oxygen saturation of the blood. The word epidural translates to "outside the dura". The dura is the outermost lining of the meninges covering the nerves of the spinal column. The epidural space is located with a needle by the anesthesiologist, and the appropriate anesthetic medications are injected. Often, a tiny catheter is left in the epidural space, taped to the patient's low back, to allow repeated doses of the medication to be given. The catheter is removed at the end of surgery, or sometimes days later if continued epidural medications are administered for postoperative pain control. Local anesthetics, such as lidocaine or bupivicaine (brand name Marcaine), given into the epidural space, bring on sensory and motor numbness. The anesthesiologist chooses the dose and type of drug depending on the patient's age, size, height, medical condition, and the type of surgery. Following the onset of numbness from epidural anesthesia, the patient may either stay awake for surgery, or more often intravenous sedation is given to achieve a light sleep. Sometimes light general anesthesia is given to supplement epidural anesthesia (Figure 3).

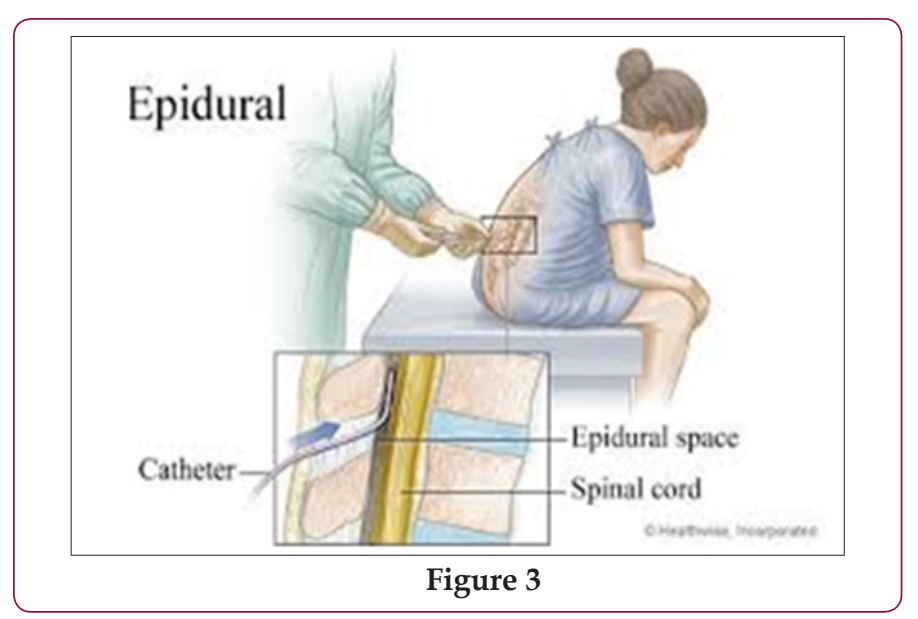

\section{Regional Anesthesia}

Regional anesthesia is the injection of local anesthetic (either lidocaine or Marcaine) near a nerve to block that nerve's function. Examples of regional anesthesia include arm blocks (axillary block, interscalene block, sub clavicular block), and leg blocks (femoral block, sciatic block, popliteal block, ankle block). An advantage of regional anesthesia blocks is that the patient may remain awake for the surgery. If desired, the anesthesia provider may administer intravenous sedation or general anesthesia in addition to the regional anesthetic, to allow the patient to sleep during the surgery-the advantage of this combined anesthetic technique is the regional anesthetic blocks all surgical pain and less sleep drugs are required.

\section{Intravenous Sedation Anesthesia}

Some minor surgical procedures (for example: breast biopsies, eyelid surgery, some hernia surgeries) can be done with the combination of local anesthesia plus intravenous anesthesia sedation. Prior to beginning anesthesia, the anesthesiologist places monitors of blood pressure, electrocardiogram, pulse and oxygen saturation of the blood. The anesthesiologist is present for the entire surgery, and administers intravenous sedatives as required for the patient's comfort and the surgeon's needs. If the sedation is deep enough, the intravenous sedation will be termed general anesthesia. While the patient is sedated, the surgeon usually injects local anesthetics into the surgical site to block both surgical and post operative pain. Vigilance by an anesthesiologist during intravenous sedation is also known as Monitored Anesthesia Care, or MAC.

\section{Pediatric Anesthesia}

Because the separation of a young child from his or her parents can be one of the most distressing aspects of the perioperative experience, many children benefit significantly from oral preoperative sedation with midazolam. This relatively pleasant-tasting liquid is given by mouth about twenty minutes prior to the start of the anesthetic. Although the midazolam rarely causes children to fall asleep, it does reduce anxiety dramatically, allowing for a much smoother separation from parents. It also tends to cause a wonderful short term amnesia, so that the children often have no recollection of separating from their parents, or even of going to the operating room. Although the initial anesthetic is usually administered via an intravenous infusion in adult patients, this approach requires starting an IV while the patient is still awake. This technique would be quite unpopular with younger children. Most young children prefer to go to sleep breathing a gas, a technique known as an inhalation induction. This technique is used for almost all routine surgeries, but cannot safely be employed in certain rare situations, such as emergencies.

An inhalation induction consists of the child breathing a relatively pleasant smelling anesthetic vapor - usually sevoflurane - via a facemask for approximately 30 to 60 seconds. The child loses consciousness while breathing the gas, and the IV can then be started painlessly. Generally, the child continues to breath the gas throughout the duration of the surgery, either via the facemask or an endotracheal tube, depending on the duration and type of surgery. It is this breathing of the gas which keeps the child anesthetized. At the end of the surgery, the gas is discontinued, and the child begins to awaken. Prior to awakening, children may be given either analgesics (pain medicines) or anti-emetics. The type of surgery will determine which of the many possible medications will be used for these purposes. Despite best attempts, it is important for parents to realize that children, especially those less than five years of age, often are somewhat cranky and irritable following anesthesia and 
surgery. We do our best to minimize this, but we cannot prevent it in all cases. Similarly, some children will experience postoperative nausea and vomiting despite receiving medications which are intended to prevent it [31].

\section{Conclusion}

In a recent update, Dr. Jeana Havidich, an associate professor of anesthesiology at Dartmouth-Hitchcock Medical Center in New Hampshire, presented the following preliminary data at the October 2014 American Society of Anesthesiologist convention. In data from from more than 3.2 million cases of anesthesia use between 2010 and 2013, the rate of complications decreased from 11.8 percent to 4.8 percent. The most common minor complication was nausea and vomiting (nearly 36 percent) and the most common major complication was medication error (nearly 12 percent).The death rate remained at three deaths per 10,000 surgeries/procedures involving anesthesia .Among the other findings: complication rates were not higher among patients who had evening or holiday procedures; patients older than 50 had the highest rates of serious complications; and healthier patients having elective daytime surgery had the highest rates of minor complications. If you have any questions, be sure to discuss them with your own physician anesthesiologist when you meet him or her prior to your surgical procedure.

\section{References}

1. Bergendahl H, Lonnqvist PA, Eksborg S (2006) Clonidine in paediatric anaesthesia: review of the literature and comparison with benzodiazepines for anesthetic premedication. Acta Anaesthesiol Scand 50 (2): 135-143.

2. Dahmani S, Brasher C, Stany I, Golmard J, Skhiri A, et al. (2010) Premedication with clonidine is superior to benzodiazepines: A meta analysis of published studies. Acta Anaesthesiol Scand 54(4): 397-402.

3. Rosenbaum Z, Kain ZN, Larsson P, Lonnqvist PA, Wolf AR, et al. (2009) The place of premedication in pediatric practice. Paediatr Anaesth 19(9): 817-828.

4. Cox RG, Nemish U, Ewen A, Crowe MJ (2006) Evidence-based clinical update: does premedication with oral midazolam lead to improved behavioural outcomes in children? (PDF). Can J Anaesth 53(12): 12131219.

5. Bozkurt P (2007) Premedication of the pediatric patient - anesthesia for the uncooperative child. Curr Opin Anesthesiol 20(3): 211-215.

6. Naguib M, Gottumukkala V, Goldstein PA ( 2007) Melatonin and anesthesia: a clinical perspective. J Pineal Res 42(1): 12-21.

7. Mencia SB, Lopez-Herce JC, Freddi N (2007) Analgesia and sedation in children: practical approach for the most frequent situations (PDF). J Pediatr(Rio J) 83(2): 71-82.

8. Manyande Anne, Cyna Allan M, Yip Peggy, Chooi Cheryl (2009) Nonpharmacological interventions for assisting the induction of anaesthesia in children. The Cochrane Database of Systematic Reviews 8(3): CD006447.

9. Hewer CL (1937) The Stages And Signs Of General Anaesthesia. BMJ 2(3996): 274-276.
10. Booth AW, Vidhani K, Lee P, Thomsett C (2017) Spontaneous Respiration using Intravenous anaesthesia and Hi-flow nasal oxygen (STRIVE Hi) maintains oxygenation and airway patency during management of the obstructed airway: an observational study. BJA: British Journal of Anaesthesia 118(3): 444-451.

11.S Contractor, JG Hardman (2006) Injury During Anaesthesia Continuing Education in Anaesthesia. Critical Care \& Pain 6(2): 67-70.

12. PN Nair, E White (2014) Care of the Eye During Anaesthesia and Intensive Care. Anaesthesia and Intensive Care Medicine 15(1): 40-43.

13. Robert K, Stoelting, Ronald D Miller Basics of Anesthesia. $5^{\text {th }}$ Edition Authors.

14. English W (2002) Post-operative shivering, causes, prevention and treatment (letter). Update in Anaesthesia 69(5): 438-532.

15. Anesthetic Routines: The Anesthesiologist's Role in GI Recovery and Postoperative Ileus.

16. Lagasse RS (2002) Anesthesia safety: model or myth? A review of the published literature and analysis of current original data. Anesthesiology 97(6): 1609-1617.

17. Arbous MS, Meursing AE, Van Kleef JW, De Lange JJ, Spoormans HH, et al. (2005) Impact of anesthesia management characteristics on severe morbidity and mortality. Anesthesiology 102(2): 257-268.

18. Nkansah PJ, Haas DA, Saso MA (1997) Mortality incidence in outpatient anesthesia for dentistry in Ontario. Oral Surg Oral Med Oral Pathol Oral Radiol Endod 83(6): 646-651.

19. Engelhart T, Webster NR (1999) Pulmonary aspiration of gastric contents in anaesthesia (PDF). Br J Anaesth 83(3): 453-460.

20. Parker RB (1956) Maternal Death from Aspiration Asphyxia. BMJ 2(4983): 16-19.

21. Dewachter P, Mouton-Faivre C, Emala CW (2009) Anaphylaxis and Anesthesia: Controversies and New Insights. Anesthesiology 111(5): 1141-1150.

22. Cooper JB, Newbower RS, Long CD, McPeek B (1978) Preventable anesthesia mishaps: a study of human factors. Anesthesiology 49(6): 399-406.

23. Beecher HK, Todd DP (1954) A Study of the Deaths Associated with Anesthesia and Surgery : Based on a Study of 599,548 Anesthesias in Ten Institutions 1948-1952, Inclusive. Ann Surg 140(1): 2-34.

24.Guadagnino C (2000) Improving anesthesia safety Narberth Pennsylvania: Physician's News Digest Inc 126(4): 1312-1320.

25. Stoelting RK (2010) Foundation History Indianapolis IN: Anesthesia Patient Safety Foundation.

26. Cottrell JE (2003) Uncle Sam, Anesthesia-Related Mortality and New Directions: Uncle Sam Wants You!. ASA Newsletter 47(7): 809-817.

27. Bronwen Jean Bryant, Kathleen Mary Knights (2011) Pharmacology for Health Professionals. Elsevier Australia.

28. Serpell MG, Fettes PD, Wildsmith JAW (2002) Pencil point spinal needles and neurological damage. British Journal of Anaesthesia 89(5): 800-801.

29. Corning J, LNY, Med J (1885) 'Classical File', Survey of Anesthesiology.

30. Bier A, Versuche Uber, Cocainisirung Des, Ruckenmarkes, Deutsch et al. (1899) 'Classical File', Survey of Anesthesiology 51: 361-378.

31. Bob Dylan (2017) A medical-legal mystery which blends the science and practice of anesthesiology with unforgettable characters, a page-turning plot. 
(c) (i) This work is licensed under Creative

Submission Link: https://biomedres.us/submit-manuscript.php

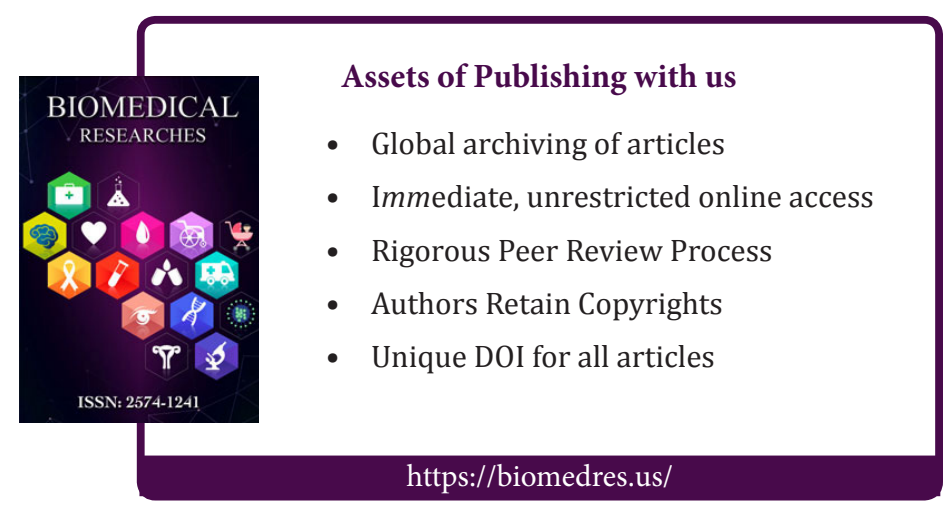

